\title{
FINE AND NONTANGENTIAL CONVERGENCE ON AN NTA DOMAIN
}

\author{
J. C. TAYLOR ${ }^{1}$
}

\begin{abstract}
The recent article by Jerison and Kenig on "Boundary behaviour of harmonic functions in nontangentially accessible domains" did not consider the relation between fine limits and nontangential limits. The results in this direction obtained by Hunt \& Wheeden [5] for Lipschitz domains are extended here to NTA domains.
\end{abstract}

In [6], Jerison and Kenig define a class of bounded domains $D$ (the so-called bounded NTA domains) for which the topological boundary is the Martin boundary of $D$ (see [6, Theorem 5.9]). Further, they show that every positive harmonic function $u$ has a nontangential limit $d \omega$-a.e. where $\omega$ is the harmonic measure of a fixed point $x_{0} \in D$. This limit is identified with $d \mu / d \omega$ if $\mu$ is a positive measure on $\partial D$ that corresponds to $u$. Their proof involves a classical maximal function argument.

For Lipschitz domains these results were obtained earlier by Hunt and Wheeden [5]. They also showed [5, Theorem 5.5] that for $u$ a positive harmonic function, $u$ has a semifine limit at a boundary point $b$ if and only if $u$ has a nontangential limit at $b$. In addition, they proved [5, Theorem 5.7] that for any function $u$ on a Lipschitz domain, if it has nontangential limits at each point of $E \subset \partial D$ then $d \omega$-a.e. it has the same fine limits on $E$ (here $\omega$ is the harmonic measure of a fixed point $x_{0}$ ). These results extended earlier work of Brelot and Doob [1] for the half space $\mathbf{R}^{n} \times \mathbf{R}^{+}$.

The main purpose of this note is to complement the work of Jerison and Kenig by establishing these additional results for all NTA domains. The usual method [1] by which it is shown that the existence of nontangential limits implies the existence of fine limits a.e. works for NTA domains. As a result, the method used by Brelot and Doob [1] to prove a local Fatou theorem from the Fatou-Naïm-Doob theorem can be applied to NTA domains (see Theorem 5.1).

It will be assumed without further comment that the value of a constant $C$ (say) can change from one use to another. If $E$ is a set $E^{c}$ denotes its complement. For the definition of potential theoretic concepts the reader is referred to Helms [4].

1. Some properties of NTA domains. A bounded domain $D \subset \mathbf{R}^{n}$ is said to be $\left(M, r_{0}\right)=$ nontangentially accessible (or NTA) [6] if there exists $M$ and $r_{0}$ such that the following three conditions are satisfied:

Received by the editors March 16, 1983.

1980 Mathematics Subject Classification. Primary 31B25; Secondary 60J50.

Key words and phrases. Fine convergence, NTA-domain.

'Aided by NSERC operating grant No. A-3108. 
(1) (The corkscrew condition). If $r<r_{0}$ and $q \in \partial D$, then there is a point $a=a(r, q) \in D$ such that (i) $r M^{-1}<|a-q|<r M$ and (ii) $\operatorname{dist}(a, \partial D)>r M^{-1}$.

(2) The complement of $D$ satisfies (1).

(3) (Harnack chain condition). If $x_{1}, x_{2} \in D$ with $\operatorname{dist}\left(x_{i}, \partial D\right) \geqslant \varepsilon$ and $\left|x_{1}-x_{2}\right|<$ $2^{k} \varepsilon$, then there are $M k$ balls $B_{j} \subset D$ such that

(i) $x_{1}$ is the centre of $B_{1}$ and $x_{2}$ is the centre of $B_{M k}$,

(ii) $B_{j} \cap B_{j+1} \neq \varnothing, 1 \leqslant j \leqslant M k-1$,

$$
\begin{gathered}
M^{-1} \operatorname{diam} B_{j} \leqslant \operatorname{dist}\left(B_{j}, \partial D\right) \leqslant M \operatorname{diam} B_{j} \quad \text { and } \\
\operatorname{diam} B_{j} \geqslant M^{-1} \operatorname{dist}\left(x_{i}, \partial D\right) \quad(i=1,2) .
\end{gathered}
$$

If $x \in \mathbf{R}^{n}$ let $B(x ; r)=\{y|| x-y \mid<r\}$ and let $\Delta(q ; r)=B(q ; r) \cap \partial D$ if $q \in \partial D$. For $x \in D$ let $\omega(x, A)$ be the harmonic measure of $A \subset \partial D$ associated with $x$, i.e. $\omega(-, A)$ is the solution of the Dirichlet problem with boundary value $1_{A}$.

REMARK 1. The Harnack chain condition has the following immediate consequence. Let $A \subset D$ and assume $\operatorname{dist} A \leqslant C_{1} \operatorname{dist}(A, \partial D)$. Then, for any positive harmonic function $u$ on $D$ there is a constant $C_{2}$ such that $u(x) \leqslant C_{2}(y)$ for all $x, y \in A$.

2. If $D$ is an $\left(M, r_{0}\right)$-NTA domain and $T(x)=\lambda x+a$ with $\lambda>0$, then it is clear that $T(D)$ is an $\left(M, \lambda r_{0}\right)$-NTA domain.

3. If $M^{\prime}>M$ and $r_{0}^{\prime}<r_{0}$, an $\left(M, r_{0}\right)$-NTA domain is also an $\left(M^{\prime}, r_{0}^{\prime}\right)$-NTA domain. It will be assumed from now on that $M \geqslant 1 \geqslant r_{0}$ unless otherwise specified.

It will be useful in what follows to make use of the following consequence of the Boundary Harnack Principle [6, 5.1].

Proposition 1.1. Let $D$ be an $\left(M, r_{0}\right)-N T A$ domain with $0 \in \partial D$ and not contained in $B(0 ; 2)$. Let $c<r_{0} / 2\left(2+M^{2}\right)$ and set

$$
A=\{x|\operatorname{dist}(x, \partial D) \geqslant c / 2,| x \mid \leqslant 3 / 2\} .
$$

Let $u, v>0$ be harmonic on $D$ and vanish continuously at $\partial D \backslash B(0 ; 1 / 2)$. Let $a \in A$. Then there is a constant $C=C\left(M, r_{0}\right)$ such that

$$
1 / C \leqslant u(x) v(a) / u(a) v(x) \leqslant C \text { for all } x \in D,|x|=1 .
$$

Proof (for the reader's convenience). The corkscrew condition implies $A \neq \varnothing$. For $x \in A$ the Harnack chain condition shows that $(*)$ is satisfied.

If $|x|=1$ and $x \notin A$, then there is a point $q \in \partial D$ with $|x-q|<c$. Since $1-c>M^{2} c+1 / 2$ (note that $r_{0} \leqslant 1$ ) it follows that $B\left(q ; M^{2} c\right) \cap B(0 ; 1 / 2)=\varnothing$. Let $a^{\prime}=a(c M, q)$ (see the corkscrew condition). Then $\operatorname{dist}\left(a^{\prime}, \partial D\right) \geqslant c$ and $a^{\prime} \in A$ since $\left|a^{\prime}-q\right|<c M^{2}$.

It then follows from $[6,4.10]$ that $(*)$ is satisfied for all $x \in D,|x|=1$.

Proposition 1.2. Let $D$ and $A$ be as in Proposition 1.1. Let $u>0$ be harmonic on $D$ and vanish continuously at $\partial D \backslash B(0 ; 1 / 2)$. Let $a \in A$. Then there is a constant $C=C\left(M, r_{0}\right)$ such that

$$
u(x) \leqslant C u(a) \text { for all } x \in D,|x|=1 .
$$


Proof. The argument used to prove Proposition 1.1 applies. One takes $a^{\prime}=$ $a\left(q, c M^{2} / 2\right)$ and instead of $[6,4.10]$ one uses $[6,4.4]$.

The proof of Lemma 4.1 in [6] shows that there are barriers (see [4] for definition) at any boundary point $b$ of $D$ that vanish at $b$ in a uniform fashion. An immediate consequence of this fact is the next result.

Proposition 1.3. Let $D$ be an $\left(M, r_{0}\right)$-NTA domain with $0 \in \partial D$ and not contained in $B(0 ; 2)$. Let $h$ denote the harmonic function on $B(0 ; 1) \cap D$ with boundary values 1 on $\partial B(0 ; 1) \cap D$ and 0 elsewhere.

Let $0<c<1$. If $\varepsilon>0$, then there exists $\delta=\delta\left(\varepsilon, c, M, r_{0}\right)$ such that $h(x)<\varepsilon$ if $\operatorname{dist}(x ; \bar{B}(0 ; c) \cap \partial D)<\delta$.

Proof. Let $2 r_{1}=\min \left\{r_{0}, 1-c\right\}$. It follows from [6, 4.1] that $h(x) \leqslant M\left(|x-q| r_{1}\right)^{\beta}$ if $|x-q|<r$ and $|q| \leqslant c, q \in \partial d$.

Corollary 1.4 (CF. [6, 4.2]). Let $1<c_{1}<2$ and $q \in \partial D$. Then, there is a constant $C_{2}$ with $\omega\left(x, \Delta\left(q, c_{1} r\right)\right) \geqslant C_{2}$ if $x \in \bar{B}(q ; r) \cap D$ as long as $r<\min \left\{1 / C_{1}, r_{0}\right\}$.

Proof. It follows (by scaling) from Proposition 1.3 that $\omega\left(x, \Delta\left(q, c_{1} r\right)\right) \geqslant 1 / 2$ if $\operatorname{dist}(x ; \bar{B}(q ; r) \cap \partial D)<c_{1} r \delta$ where $\delta=\delta\left(1 / 2,1 / C_{1}, M, r_{0}\right)$. The Harnack chain condition, applied to $\bar{B}(q ; r) \cap\left\{x \in D \mid \operatorname{dist}(x, \partial D) \geqslant c_{1} r \delta\right\}$, completes the argument.

Proposition 1.5. Let $D$ be an $\left(M, r_{0}\right)-N T A$ domain with $0 \in \partial D$ and not contained in $B(0 ; 2)$. Let $k$ denote the harmonic function on $D \cap \bar{B}(0 ; 1)^{c}$ with boundary values 1 on $\partial B(0 ; 1) \cap D$ and 0 elsewhere. Let $1<c<2$. If $\varepsilon>0$ then there exists $\delta=$ $\delta\left(\varepsilon, c, M_{1} r_{0}\right)$ such that $k(x)<\varepsilon$ if $\operatorname{dist}\left(x, \bar{B}(0 ; c)^{c} \cap \partial D\right)<\delta$.

Proof. It is similar to the proof of Proposition 1.3.

2. A bubble set is not minimally thin. Let $D$ be an NTA domain.

Definition 2.1. Let $\alpha>0$ and $b \in D$. The set $\Gamma_{\alpha}(b)=\{x \in D|| x-b \mid<$ $(1+\alpha) \operatorname{dist}(x, \partial D)\}$ is called a nontangential region (or corkscrew) at $b$ (see $[6,5.6])$. Let $\Gamma_{\alpha}(b ; h)=\Gamma_{\alpha}(b) \cap B(b ; h)$.

A function $u$ on $D$ has nontangential limit $\lambda$ at $b$ if $u(x) \rightarrow \lambda$ as $x \rightarrow b$ through any corkscrew $\Gamma_{\alpha}(b)$ for which $b \in \overline{\Gamma_{\alpha}(b)}$.

Definition 2.2. A sequence $\left\{x_{n}\right\}$ converges to $b \in \partial D$ nontangentially if there exists a constant $C>1$ and a sequence $\left\{r_{n}\right\} \subset \mathbf{R}_{+}$converging to zero such that

(i) $r_{n} C^{-1}<\left|x_{n}-b\right|<r_{n} C$,

(ii) $\operatorname{dist}\left(x_{n}, \partial D\right)>r_{n} C^{-1}$.

Lemma 2.3. $A$ function $u$ on $D$ has nontangential limit $\lambda$ at $b \in \partial D$ if and only if $\lambda=\lim _{n \rightarrow \infty} u\left(x_{n}\right)$ whenever $\left\{x_{n}\right\}$ converges to $b$ nontangentially.

Definition 2.4. A bubble set at $b \in \partial D$ is a set $B=\cup_{n=1}^{\infty} B_{n}$, where $B_{n}=$ $B\left(x_{n} ; \gamma r_{n} C^{-1}\right)$ with $0<\gamma<1$ and $\left\{x_{n}\right\}$ a sequence converging to $b$ nontangentially.

Notation. If $u \geqslant 0$ is superharmonic on $D$, let $R_{E} u(x)$ denote $\inf \{v(x) \mid v \geqslant 0$, superharmonic on $D, v \geqslant u$ on $E$ \}. (It is called the réduite of $u$ relative to $E$ and is denoted by $R_{E}^{u}$ in [4].) 
LEMMA 2.5. Let $\left\{x_{n}\right\}$ converge to $b \in \partial D$ nontangentially and let $B_{n}=B\left(x_{n} ; \gamma r_{n} C^{-1}\right)$ be the nth ball in an associated bubble set.

Assume $B^{\prime} \cap B_{n} \neq \varnothing$ where $B^{\prime}$ is a ball $\subset D$ and $M^{-1} \operatorname{diam} B^{\prime} \leqslant \operatorname{dist}\left(B^{\prime}, \partial D\right) \leqslant$ $M$ diam $B^{\prime}$. Then there is a constant $N=N(\gamma, C, M)>0$ such that

$$
R_{B_{n}} 1(x) \geqslant N \text { if } x \in B^{\prime} \text {. }
$$

Proof. Let $r^{\prime}$ be the radius of $B^{\prime}$. Then $M^{-1} 2 r^{\prime} \leqslant \operatorname{dist}\left(B^{\prime}, \partial D\right) \leqslant\left|x_{n}-b\right|+$ $\gamma r_{n} / C \leqslant r_{n}\left\{C+\gamma C^{-1}\right\}$. Translate the centre of $B^{\prime}$ to the origin and scale by $1 / r^{\prime}$. The image $\tilde{B}_{n}$ of $B_{n}$ is a ball of radius $\gamma r_{n} C^{-1} / r^{\prime} \geqslant R>0$, where

$$
R=2 \gamma M^{-1} C^{-1}\left\{C+\gamma C^{-1}\right\}^{-1}=R(\gamma, C, M) .
$$

Hence, to prove the lemma it suffices to solve the following problem. Let $W=B(0 ; 1+\eta)$ and let $B_{t}=B\left(t e_{1} ; R\right)$ with $0 \leqslant t \leqslant R+1$. Is there a constant $N=N(\eta, R)$, independent of $t$, such that if $p$ is the equilibrium or capacitory potential (see [4] for definition) on $W$ of $B_{t} \cap W$ then $p(x) \geqslant N$ for all $x,|x| \leqslant 1$ ? It will be assumed that $\eta \leqslant \min \left\{M^{-1}, 1 / 3 R\right\}$. Let $q_{t}$ be the equilibrium potential on $W$ of $B\left(t e_{1} ; \eta / 2\right)$. For any fixed value of $t$ it has a minimum on $\bar{B}(0 ; 1)$. It suffices therefore to show that there is a finite number of values of $t$, say, $t_{1}, \ldots, t_{m}$ where $m=m(n, R)$ such that $|x| \leqslant 1 \Rightarrow p(x) \geqslant q_{t_{i}}(x)$ for some $i=1, \ldots, m$. To see this let $t_{1}=1+\eta / 2, t_{k+1}=t_{k}-2 R+\eta$.

Corollary 2.6. Let $D$ be an $\left(M, r_{0}\right)$-NTA domain. Let $\left\{x_{n}\right\}$ converge to $b \in \partial D$ nontangentially and let $B_{n}=B\left(x_{n} ; \gamma r_{n} C^{-1}\right)$ be the nth ball in an associated bubble set.

Let $r<r_{0}$ and let $2 r_{n} C<r$ for $n \geqslant n(r)$. If $u>0$ is harmonic on $D$ and $w=R_{B_{n}} 1$, then there is a constant $C_{1}=C_{1}\left(M, r_{0}, \gamma, C\right)$ such that

$$
C_{1}^{-1} \leqslant \frac{u(x) w\left(x_{n}\right)}{u\left(x_{n}\right) w(x)} \leqslant C_{1}
$$

for all $x \in D,|x|=2 r_{n}\left(C+\gamma C^{-1}\right)$ providing $u$ vanishes continuously at $\partial D \backslash\{b\}$.

Proof. Let $C$ be the constant in Definition 2.2 associated with $\left\{x_{n}\right\}$. Let $\lambda^{-1}=2 r_{n}\left(C+\gamma C^{-1}\right)$. Set $T(x)=\lambda(x-b)$ and consider the image of $D$ under $T$. If the constant $c$ of Proposition 1.1 is taken less than $1 /\left(2 C^{2}+2 \gamma\right)$, then $T\left(x_{n}\right) \in A$ $=\{x|\operatorname{dist}(x, \partial T(D)) \geqslant c / 2| x \mid, \leqslant 3 / 2\}$.

The argument of Proposition 1.1 applies once certain observations have been made. Let $x_{1}, x_{2} \in D$ and let $\left(B_{i}^{\prime}\right)$ be a Harnack chain from $x_{1}$ to $x_{2}$. Then there is a constant $C$ such that $u\left(x_{1}\right) \leqslant C u\left(x_{2}\right), w\left(x_{1}\right) \leqslant C w\left(x_{2}\right)$ where $u>0$ is harmonic on $D$. To see this let $B_{j}^{\prime}$ be the first ball to meet $B_{n}$ and $B_{l}^{\prime}$ be the last ball to meet $B_{n}$. For all positive harmonic functions $v$ on $D \backslash B_{n}$, there are constants $C_{1}, C_{2}$ such that $v\left(x_{1}\right) \leqslant C_{1} v(x) \leqslant C_{1}^{2} v\left(x_{1}\right)$ for all $x \in B_{j-1}^{\prime}$ and $v\left(x_{2}\right) \leqslant C_{2} v(y) \leqslant C_{2}^{2} v\left(x_{2}\right)$ for all $y \in B_{l+1}^{\prime}$. Replacing $B_{j+1}^{\prime}, \ldots, B_{l-1}^{\prime}$ by $B_{n}$ shows that

$$
w\left(x_{1}\right) \leqslant C_{1} w(x) \leqslant C_{1} \leqslant C_{1} N^{-1} w(y) \leqslant C_{2} C_{1} N^{-1} w\left(x_{2}\right) .
$$

Consequently, the growth of $w$ is controlled by Harnack chains in the same fashion as the growth of any positive harmonic function. 
Jerison and Kenig proved that the Martin Boundary of $D$ (see [4] for definition) can be identified with the Euclidean boundary [6, Theorem 5.9]. Furthermore, all boundary points are minimal (see [4] for definition) in view of [6, Theorem 5.10]. It states that to each point $b \in \partial D$ there corresponds a positive harmonic function $K_{b}(x)$, normalized by requiring $K_{b}\left(x_{0}\right)=1$ for a fixed point $x_{0} \in D$. This "Poisson" kernel represents all positive harmonic functions $u$ on $D$. More precisely, to each positive harmonic function $u$ on $D$ there is a unique positive Borel measure $\mu$ with $u(x)=\int K_{b}(x) \mu(d b)$ for all $x \in D$. Consequently, $K_{b}$ is a minimal harmonic function (i.e. if $0 \leqslant u \leqslant K_{b}$ and $\Delta u=0$, then $u=\lambda K_{b}$ for some $\lambda \geqslant 0$ ).

Definition 2.7. $A$ set $E \subset D$ is (minimally) thin at $b \in \partial D$ if there is a superharmonic function $v$ on $D$ distinct from $K_{b}$ such that

(i) $v=K_{b}$ on $E$ and

(ii) $v \leqslant K_{b}$ i. e. if $R_{E} K_{b} \neq K_{b}$.

It is well known that the union of two sets that are thin at $b$ is also thin at $b$, since a set $E$ is thin at $b$ if and only if $K_{b}$ is dominated on $E$ by a potential (cf. [10]). Hence, the sets whose complements are thin at $b$ form a filter $F(b)$.

DEFInItion 2.8. A function $\mu$ on $D$ has a fine limit at $b$ if it has a limit along the filter $F(b)$.

The theorem of Fatou-Naïm-Doob $[2,9,10]$ implies that if $u, h$ are any two positive harmonic functions on $D$ then $\nu$-a.e. $u / h$ has fine limit at $b \in \partial D$ equal to $(d \mu / d \nu)(b)$ where $\mu$ and $\nu$ are the measures such that $u(x)=\int K_{b}(x) \mu(d b)$ and $h(x)=\int K_{b}(x) \nu(d b)$.

LEMMA 2.9 (BRELOT - DOOB). Let $b \in \partial D$ and assume that any bubble set at $b$ is not thin at $b$. Let $u, h$ be positive harmonic functions on $D$. Then if $\lambda$ is the fine limit at $b$ of $u / h$ it is the nontangential limit of $u / h$ at $b$.

Proof (CF. [1, ThÉrème 3]). Let $\eta$ be a nontangential cluster value of $u / h$ at $b$. Then there is a sequence $\left\{x_{n}\right\}$ converging nontangentially to $b$ such that $\eta=$ $\lim _{n \rightarrow \infty} u\left(x_{n}\right) / h\left(x_{n}\right)$.

Let $\varepsilon>0$. By Harnack's inequality there is a bubble set $B$ associated with $\left\{x_{n}\right\}$ such that $|u(x) / h(x)-\eta|<\varepsilon$ for all $x \in B_{n}$ and $n$ sufficiently large. Since the bubble set is not thin at $b$, it meets every set in $F(b)$ and so $\lambda=\eta$.

Consequently, in order to deduce the existence of nontangential limits from the Theorem of Fatou-Naïm-Doob it suffices to verify the following result.

Proposition 2.10. Let $B=\cup_{n=1}^{\infty} B_{n}$ be a bubble set at $b \in \partial D$. Then $B$ is not thin at $b$.

Proof. If $B$ is thin at $b$, then there is a potential $p$ on $D$ with $p \geqslant K_{b}$ on $B$. Let $u_{n}=R_{B_{n}} K_{b}$. The existence of $p$ is impossible if there is a constant $C>0$ with $u_{n}\left(x_{0}\right) \geqslant C$, where $x_{0}$ is the normalising point for which $K_{b}\left(x_{0}\right)=1$.

In view of Harnack's inequality applied to $K_{b}$ on $\bar{B}_{n}$ it follows that there is a constant $C$ with $C^{-1} \leqslant u_{n}(x) / u_{n}\left(x_{n}\right) \leqslant C$ where $x_{n}$ is the centre of $B_{n}$ and $x \in B_{n}$. It follows from Corollary 2.6 that $u_{n}(x) \geqslant C K_{b}(x)$ for all $x \in D,|x|=$ $2 r_{n}\left(C+\gamma C^{-1}\right)$. The maximum principle implies that $u_{n}\left(x_{0}\right) \geqslant C>0$. 
Corollary 2.11. Let $u, h>0$ be harmonic on $D$ and "Poisson" integrals of the measures $\mu, \nu$ (respectively). Then $u / h$ has nontangential limit $(d \mu / d \nu)(b) \nu$-a.e. In particular, $u$ has nontangential limit $(d \mu / d \omega)(b) \omega$-a.e. where $w=\omega\left(x_{0},-\right)$ is the harmonic measure corresponding to $x_{0}$.

Proof. The first statement is an immediate consequence of Proposition 2.10, Lemma 2.9 and the Theorem of Fatou-Naïm-Doob.

The second statement is a particular case of the first once it is established that the constant function 1 is represented by $\omega$. Let $\lambda$ be the measure such that $\int K_{b}(x) \lambda(d b)=1, \forall x \in D$.

The kernel function approach to the determination of the minimal functions $K_{b}$ makes it obvious that $\lambda=\omega$. However, this fact is always true and in this particular case is easily established from general considerations.

Let $f \in C(\partial D)$, set $h(x)=\int K_{b}(x) f(b) \lambda(d b)$ and $u(x)=\int f(b) \omega(x, d b)$, where $\omega(x, d b)$ is the harmonic measure corresponding to $x$. Since an NTA-domain is regular (a consequence of $[6,4.1]$ ) (see $[4]$ for definition) the ordinary boundary limit (and hence the fine limit) of $u$ at $\partial D$ is $f$. Consequently, $h-u=v$ is a bounded harmonic function with fine limit equal to zero $\lambda$-a.e. Since every positive harmonic is the (Poisson) integral of a unique positive measure on $\partial D$ this implies $v=0$. Consequently, $K_{b}(x) \lambda(d b)=\omega(x, d b)$ and, in particular, $\lambda=\omega\left(x_{0},-\right)=\omega$.

3. Semifine convergence and nontangential convergence. Fix $b \in \partial D$ and let $E \subset D$. Set $E_{k}=\left\{x \in E\left|2^{-k-1} \leqslant\right| x-b \mid \leqslant 2^{-k}\right\}$.

DeFinition 3.1. $E$ is said to be semithin at $b$ if $\lim _{k \rightarrow \infty} R_{E_{k}} K_{b}=0$.

Since $R_{A_{1} \cup A_{2}} u \leqslant R_{A_{1}} u+R_{A_{2}} u$ for any two sets $A_{i} \subset D$ and $u \geqslant 0$ superharmonic on $D$, it follows that the union of two sets that are semithin at $b$ is also semithin at $b$. Let $S(b)=\left\{E^{c} \mid E\right.$ semithin at $\left.b\right\}$.

DEFINITION 3.2. A function $f$ has semifine limit at $b$ if it converges along $S(b)$.

Proposition 3.3. A bubble set at $b$ is not semithin at $b$.

Proof. Let $B=\cup_{n=1}^{\infty} B_{n}, B_{n}=B\left(x_{n} ; \gamma r_{n} C^{-1}\right)$ where $\left\{r_{n}\right\}$ and $C$ are as in Definition 2.2. Then, each $B_{n}$ meets at most $m+1$ of the sets $\left\{x \in D\left|2^{-k-1} \leqslant\right| x-b \mid \leqslant\right.$ $\left.2^{-k}\right\}$ if $m \log 2 \geqslant[4 \log C-\log (1-r)]$. Since $R_{A_{1} \cup A_{2}} u \leqslant R_{A_{1}} u+R_{A_{2}} u$ the result follows from the fact established in the proof of Proposition 2.8: $R_{B_{n}} K_{b}\left(x_{0}\right) \geqslant C>0$.

Proposition 3.4. Let $E \subset D$ be such that $\operatorname{dist}(x, \partial D)|x-b|^{-1} \rightarrow 0$ as $|x-b| \rightarrow 0$ (i.e. let $E$ be a tangential set). Then $E$ is semithin at $b$ (the point used to define the sets $\left.E_{k}\right)$.

Proof. $E$ is a tangential set at $b$ if and only if $2^{k} \operatorname{dist}\left(E_{k}, \partial D\right) \rightarrow 0$ as $k \rightarrow \infty$. Let $\delta>0$ and let $k(\delta)$ be such that $k>k(\delta)$ implies $2^{k} \operatorname{dist}(x, \partial D)<\delta$ for all $x \in E_{k}$.

In view of Propositions 1.3 and 1.5 it is possible to choose $\delta$ so that the harmonic function $h_{k}$ on $D \cap\left\{x\left|2^{-k-2}<\right| \kappa-b \mid<2^{-k+1}\right\}$ with houndary values 1 on $\left\{B\left(b ; 2^{-k-2}\right) \cap D\right\} \cup\left\{B\left(b ; 2^{-k+1}\right) \cap D\right\}$ and 0 elsewhere is less than $\varepsilon$ on $E_{k}$.

Let $a_{k}=a\left(2^{-k}, b\right)$. Then by Proposition 1.2 there is a constant $C$ with $K_{b}(x) \leqslant$ $C K_{b}(a)$ if $|x|=2^{-k-2}$ or $|x|=2^{-k+1}$. Hence, $x \in E_{k}$ implies $K_{b}(x) \leqslant \varepsilon C K_{b}(a)$. 
Let $w_{k}(x)=w\left(x, \Delta\left(b, 2^{-k+1}\right)\right)$. It follows from Corollary 1.4 that there is a constant with $C w_{k}(x) \geqslant R_{D_{k}} 1(x)$ if $|x-b|>2^{-k+2}, D_{k}=D \cap\left\{x\left|2^{-k-1} \leqslant\right| x \mid \leqslant\right.$ $\left.2^{-k}\right\}$. Consequently,

$$
R_{E_{k}} K_{b}(x) \leqslant \varepsilon C K_{b}\left(a_{k}\right) w_{k}(x) \text { for }|x-b|>2^{-k+2} .
$$

In particular, $R_{E_{k}} K_{b}\left(x_{0}\right) \leqslant \varepsilon C K_{b}\left(a_{k}\right) w_{k}\left(x_{0}\right)$.

Let $a_{k-2}=a\left(2^{-k+2}, b\right)$. It follows from [6, 4.2] (or Corollary 1.4) that $w_{k}\left(a_{k-2}\right)$ $\geqslant C$. Also, by the Harnack chain condition, for some constant $C, K_{b}\left(a_{k}\right) \leqslant$ $c K_{b}\left(a_{k-2}\right) \leqslant C^{2} K_{b}\left(a_{k}\right)$. Scaling by $1 / r_{k}, r_{k}=\left|a_{k-2}\right|$, it follows from Proposition 1.1 that $|x-b|>2^{-k+2}$ implies $w_{k}(x) K_{b}\left(a_{k}\right) \leqslant C K_{b}(x)$. Since $K_{b}\left(x_{0}\right)=1$, the previous estimate for $R_{E_{k}} K_{b}\left(x_{0}\right)$ shows that $R_{E_{k}} K_{b}\left(x_{0}\right) \leqslant C \varepsilon$.

Proposition 3.4. Let $u, h$ be $>0$ harmonic. Then $u / h$ has a semifine limit $\lambda$ at $b \Leftrightarrow u / h$ has nontangential limit $\lambda$ at $b$.

Proof $(\Rightarrow)$. This follows from Proposition 3.3 by the argument used to prove Lemma 2.9.

$(\Leftarrow)$. Let $\lambda$ be a semifine limit value of $u / h=f$ at $b$ i.e. for every $\varepsilon>0$, $E=\{x \mid \lambda-\varepsilon<f(x)<\lambda+\varepsilon\}$ meets every set in $S(b)$. It must meet every $\Gamma_{\alpha}(b)$ for which $b \in \overline{\Gamma_{\alpha}(b)}$. Otherwise, for any such $\alpha$, there exists $r(\alpha)$ with $\Gamma_{\alpha}(b) \cap\{x \mid \lambda-\varepsilon$ $<f(x)<\lambda+\varepsilon\} \cap B(b ; r)=\varnothing$ if $r<r(\alpha)$. In this case, $2^{k} \operatorname{dist}\left(E_{k}, \partial D\right) \rightarrow 0$ as $k \rightarrow \infty$. Hence, $\lambda$ is a nontangential limit value of $f$ at $b$.

4. Nontangential convergence implies fine convergence $d \omega$-a.e. Let $D$ be a bounded $\left(M, r_{0}\right)$-NTA domain and let $F \subset \partial D$ be closed. Let $W=\cup_{b \in F} \Gamma_{\alpha}(b ; h)$.

Proposition 4.1. $W^{c}$ is thin at dw-a.e. point of $F$.

Proof (CF. [1, Thérème 8]). Assume $(1+\alpha) \operatorname{dist}(x, \partial D)<h$. If $x \notin W$ then $x \notin \Gamma_{\alpha}(b)$ for all $b \in F$. Hence, $|x-b| \geqslant(1+\alpha) \operatorname{dist}(x, \partial D)$. Let $q \in \partial D$ be such that $|x-q|=\operatorname{dist}(x, \partial D)$. Then, if $b \in F$,

$$
|q-b|+|x-q| \geqslant|x-b| \geqslant(1+\alpha)|x-q|
$$

and so $|q-b| \geqslant \alpha|x-q|$. Let $r=\operatorname{dist}(q, F)$. Then, $r \geqslant \alpha|x-q|$. It follows from [6, 4.2] that $\omega(x, \Delta(q, r / 2)) \geqslant C$ since $x=a(|x-q|, q)$ where $0<C<1$.

Let $u$ be the harmonic function with boundary value $1_{F}$. It follows from the above that if $(1+\alpha) \operatorname{dist}(x, \partial D)<h$ and $u(x)>1-C$ then $x \in W$. The theorem of Fatou-Naïm-Doob implies that $\{x \mid u(x)>1-C\}$ is a fine neighbourhood of $d \omega$-a.e. point of $F$. Since $\{x \mid(1+\alpha) \operatorname{dist}(x, \partial D)<h\}$ is a neighbourhood (hence a fine neighbourhood) of $\partial D$ the result follows.

TheOREM 4.2. Let $D$ be an NTA-domain and $f: D \rightarrow \mathbf{R}$ a function that has a nontangential limit $\varphi$ on $E \subset \partial D$. Then $d \omega$-a.e. on $E, f$ has fine limit $\varphi$.

PROOF. The argument used to prove Theorem 5.7 in [5] applies.

5. A local Fatou theorem. Let $D$ be an NTA-domain and let $W=\cup_{b \in E} \Gamma_{\alpha}(b, h)$ where $h, \alpha$ vary with $b$. Assume $u$ is harmonic on $W$ and lower bounded on each $\Gamma_{\alpha}(b, h)$. Extend $u$ trivially to $D$ by setting $u(x)=0$ if $x \in D \backslash W$. 
TheOREM 5.1 (CF. [6, THEOREM 6.4]). At dw-a.e. $b \in E$ the extended function $u$ has a nontangential limit as a function on $D$.

Proof. As usual it suffices to consider the case of $E$ closed, $\alpha$ and $h$ independent of $b \in E$ and $u>0$. Let $E_{0} \subset E$ be the set of points $b$ for which $W$ is a fine neighbourhood. $E_{0}$ can be identified with a subset of the Martin boundary $\partial D$ of $D$ and of $W[3,7]$ and the null subsets of $F_{0}$ for $d \omega$ and the measure representing 1 on $W$ coincide. Hence, the theorem of Fatou-Naïm-Doob applied to $u$ on $W$ implies that $d \omega$-a.e. $u$ has a fine limit on $D$ at the points of $E$.

The Caldéron Density Lemma (i.e. [6, 6.1]) and the proof of Lemma 2.9 imply that $u$ has nontangential limits a.e. on $E$ (note that in Lemma $2.9 u$ can be taken to be defined on a corkscrew with large $\alpha$ ).

REMARK. This proof of a local Fatou theorem avoids the problem of constructing sub-NTA domains of $D$ in neighbourhoods of boundary points of $D[6$, Theorem 3.11] which was solved by Jones [7].

\section{BIBLIOGRAPHY}

1. M. Brelot and J. L. Doob, Limites angulaires et limites fines, Ann. Inst. Fourier (Grenoble) 13 (1963), 395-415.

2. J. L. Doob, Conditional Brownian motion and boundary limits of harmonic functions, Bull. Soc. Math. France 85 (1957), 431-458.

3. K. Gowrisankaran, Extreme harmonic functions and boundary value problems. II, Math. Z. 94 (1966), 256-270.

4. L. L. Helms, Introduction to potential theory, Wiley-Interscience, New York, 1969.

5. R. A. Hunt and R. L. Wheeden, Positive harmonic functions on Lipschitz domains, Trans. Amer. Math. Soc. 147 (1970), 507-527.

6. David S. Jerison and Carlos E. Kenig, Boundary behaviour of harmonic functions in non-tangentially accessible domains, Adv. in Math. 46 (1982), 80-147.

7. Peter W. Jones, A geometric localization theorem, Adv. in Math. 46 (1982), 71-79.

8. A. Koranyi and J. C. Taylor, Fine convergence and admissible convergence for symmetric spaces of rank one, Trans. Amer. Math. Soc. 263 (1981), 169-181.

9. L. Naïm, Sur le rôle de la frontière de R. S. Martin dans la théorie du potentiel, Ann. Inst. Fourier (Grenoble) 7 (1957), 183-281.

10. J. C. Taylor, An elementary proof of the theorem of Fatou-Naïm-Doob, Canad. Math. Soc. Conf. Proceedings, vol. 1, Amer. Math. Soc., Providence, R.I., 1981, pp. 153-163.

Department of Mathematics, McGill University, 805 Sherbrooke St. West, Montreal, P.Q., CANADA H3A $2 \mathrm{~K} 6$ 